\title{
VIABILIDADE ECONÔMICA DA IRRIGAÇÃO DA CULTURA DO CAFÉ NA REGIÃO DE VIÇOSA-MG ${ }^{1}$
}

\author{
Alan Figueiredo de Arêdes ${ }^{2}$ \\ Maurinho Luiz dos Santos ${ }^{3}$ \\ José Luis dos Santos Rufino ${ }^{4}$ \\ Brício dos Santos Reis ${ }^{5}$
}

\begin{abstract}
Resumo - Este artigo teve por objetivo analisar a viabilidade econômica do emprego de diferentes alternativas tecnológicas de irrigação na cultura do café na região de ViçosaMG, caracterizada por condições hídricas consideradas favoráveis à cafeicultura. Para isso, foram analisados os parâmetros técnicos e econômicos do cultivo de café em cinco diferentes alternativas tecnológicas: produção não-irrigada com baixa produtividade; produção não-irrigada com alta produtividade; produção irrigada por gotejamento; produção fertirrigada por gotejamento; e produção irrigada por malha. Para cada uma das alternativas, obtiveram-se os indicadores econômicos Valor Presente, Taxa Interna de Retorno, Taxa Interna de Retorno Modificada e Período de Payback; realizou-se a análise de risco pelo método de Latin Hypercube. De acordo com os resultados, concluise que a produção de café irrigado é economicamente superior à produção não-irrigada mesmo em regiões tradicionalmente produtoras, como na região de Viçosa-MG, pois, além de elevar o retorno econômico e reduzir o tempo de retorno do capital investido, a cafeicultura irrigada ainda reduz o risco da atividade.
\end{abstract}

Palavras-chave: café, retorno, risco.

Recebido em: 14/02/2007 Aceito em: 06/06/2007

Doutorando em Economia Aplicada do Departamento de Economia Rural da UFV.

E-mail: aredess@yahoo.com.br

3 Professor do Departamento de Economia Rural da UFV.

4 Pesquisador da Embrapa Café.

5 Professor do Departamento de Economia Rural da UFV. 


\section{Introdução}

Mesmo com o significativo crescimento da irrigação das lavouras de café no Brasil, não existem informações concretas sobre a viabilidade econômica desse processo para responder às conquistas tecnológicas obtidas ao longo do tempo, havendo controvérsias sobre a viabilidade do uso da irrigação.

Em áreas de fronteira agrícola, que possuem temperatura, altitude e solos adequados ao cultivo de café, mas elevados déficits hídricos, os resultados empíricos apontam no sentido da viabilidade econômica da irrigação, mesmo porque a condução das lavouras seria inviável sem ela. Nas regiões produtoras tradicionais, onde as práticas são feitas de forma suplementar, a viabilidade econômica dessa prática é muito discutida e carente de estudos. Autores como Silva et al. (2003) afirmam que não existe resposta definitiva sobre sua viabilidade em regiões úmidas, o que justifica pesquisas acerca da irrigação do cafeeiro nessas áreas.

Estudos têm demonstrado que mesmo em regiões com condições hídricas favoráveis ocorre ganho de produtividade na cafeicultura irrigada, sendo esse sistema tecnicamente viável, como é observado por Mantovani (2003) e Coelho et al. (2006). Nessas áreas cafeeiras tradicionais, o uso dos sistemas tem sido direcionado para contornar a não-regularidade de chuvas e ocorrência de veranicos, especialmente nas fases de floração e granação (ITEM, 2000).

No entanto, para que a implementação dos sistemas seja economicamente viável, é necessário que os benefícios gerados com o uso da irrigação sejam positivos e superem os retornos provenientes da cafeicultura nãoirrigada, ou seja, que o ganho monetário pela elevação da produtividade compense a elevação do custo de produção ocasionada pela implantação e operacionalização dos sistemas de irrigação.

Nesse sentido, justifica-se a análise da viabilidade econômica da utilização das alternativas tecnológicas de produção de café com os sistemas de 
Alan Figueiredo de Arêdes, Maurinho Luiz dos Santos,

José Luis dos Santos Rufino \& Brício dos Santos Reis

irrigação do cafeeiro em regiões tradicionais, caracterizadas por condições hídricas favoráveis à cafeicultura, uma vez que, com adoção das técnicas de irrigação, espera-se a elevação da rentabilidade gerada a partir da utilização desses sistemas.

Assim, realizou-se um estudo de caso sobre a viabilidade econômica do emprego de alternativas tecnológicas de irrigação na produção de café na região de Viçosa-MG, localizada na Zona da Mata mineira, que pertence a uma região úmida tradicionalmente produtora de café. A escolha desse local para pesquisa deve-se à importância econômica do cultivo do café para o município e ao fato de a região apresentar um nível pluviométrico favorável à cafeicultura, possibilitando a concretização do trabalho. Esta pesquisa contribuirá diretamente para a tomada de decisão no tocante ao emprego de tais técnicas pelos cafeicultores dessas áreas.

\section{Metodologia}

\subsection{Retorno e risco em projetos}

Segundo Buarque (1991), a análise de projetos é feita pela ótica econômica e privada, sendo a primeira entendida como a análise dos efeitos do projeto sobre a economia global na realização do bem coletivo. Já a análise privada considera a geração dos benefícios financeiros gerados pelos projetos para a satisfação empresarial. Assim, a primeira análise apresenta os benefícios e custos do projeto em valores econômicos e a segunda considera os valores em termos de mercado. Buarque (1991) salienta que existe ainda a ótica social, em que se promove o estudo dos efeitos do projeto na distribuição dos benefícios e custos gerados sobre as diferentes classes sociais.

Dentre as etapas de elaboração de projetos, destacam-se as análises quantitativas e qualitativas que resultarão no fluxo de caixa do projeto. A correta construção do fluxo é de suma importância, uma vez que os 
indicadores de rentabilidade e risco do projeto são derivados dele, ou seja, das entradas e saídas de numerários ocorridas durante o período de sua vigência. No que se refere aos critérios econômicos de viabilidade de projetos, merecem destaque o Valor Presente Líquido (VPL), a Taxa Interna de Retorno (TIR) e o Período de Payback (PP).

Conforme Ross et al. (1998), as principais vantagens e desvantagens dos critérios de avaliação econômica de projetos VPL, TIR e PP são:

- Valor Presente Líquido (VPL): critério de decisão preferível, não apresentando deficiências sérias.

- Taxa Interna de Retorno (TIR): relacionado com o VPL e de fácil entendimento e transmissão. No entanto, apresenta deficiências, como: possibilidade de obtenção de respostas múltiplas e geração de decisões incorretas em projetos mutuamente excludentes.

- Período de Payback (PP): fácil entendimento, considera as incertezas de fluxos de caixa mais longos e funciona como um ponto de equilíbrio para o investimento. A desvantagem do critério está em basear-se em um período de tempo-limite, desconsiderar fluxos de caixa além do tempo-limite, ser viesado na análise de projetos com vida útil elevada e não considerar o valor do dinheiro no tempo ${ }^{6}$.

Outro critério indicado para avaliação de projetos é o da Taxa Interna de Retorno Modificada (TIRM):

- Taxa Interna de Retorno Modificada (TIRM): é a TIR quando se consideram o custo de investimento e os juros de reinvestimento dos fluxos de caixa. Considera o verdadeiro custo de oportunidade do capital investido, visto que a TIR considera

\footnotetext{
Existe a possibilidade de considerar o valor do dinheiro no tempo. Nesse caso, o Período de Payback (PP) é denominado Período de Payback Descontado (PPD). Já o VPL, TIR e TIRM sempre são calculados considerando-se o tempo.
} 
Alan Figueiredo de Arêdes, Maurinho Luiz dos Santos,

José Luis dos Santos Rufino \& Brício dos Santos Reis

reinvestimento a própria TIR; assim, a TIRM é melhor indicador que a TIR.

A análise de projetos inclui ainda os riscos do investimento, visto que todo projeto está sujeito a riscos e incertezas (Buarque, 1991), de tal forma que as incertezas existem quando determinados eventos futuros não são conhecidas. Por outro lado, riscos existem quando probabilidades de realização de eventos futuros são conhecidos, sendo os riscos então quantificados (Woiler e Mathias, 1996).

Para Woiler e Mathias (1996), riscos são devidos principalmente à grande quantidade de informação contida em projetos de investimento e a utilização de valores não reais. Segundo os autores, riscos podem ser classificados como internos e externos, sendo os primeiros provenientes de fontes endógenas e passíveis de influências empresariais, ao passo que os riscos externos são oriundos de fontes exógenas e as empresas possuem poucos meios para contorná-los. Exemplo de riscos internos é a capacidade de administração do gerente e seu processamento correto de informações. No tocante aos riscos externos, tem-se como exemplo o risco de mercado e o natural (climático, biológico, químico e outros).

Thiry-Cherques (2004) sugere as seguintes opções para minimização dos riscos: exaustiva análise das influências das externalidades no projeto; obtenção de informações históricas recentes no setor onde o projeto será concretizado; e levantamento de dados de mercado e de informações comerciais. Woiler e Mathias (1996) argumentam que a análise de risco é aperfeiçoada pelo emprego das simulações de Monte Carlo, baseadas na aleatoriedade dos valores das variáveis.

Diante do risco, os indivíduos podem apresentar três diferentes comportamentos: ser avesso, propenso ou indiferente (Gitman, 1997; Varian, 1993; Debertin, 1986; Pindyck e Rubinfeld, 2006). A Figura 1 demonstra em forma gráfica os diferentes comportamentos. 


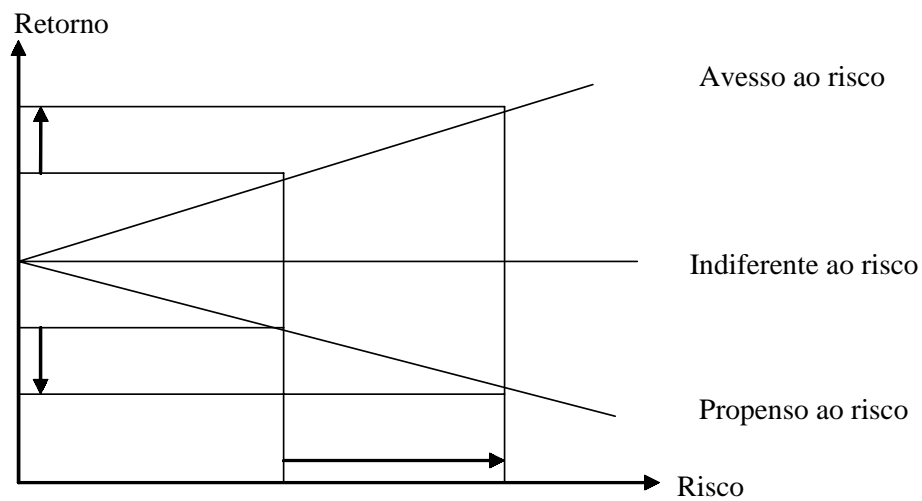

Figura 1 - Preferências e comportamentos em relação ao risco. Fonte: Gitman (1997)

Pela Figura 1, se o indivíduo for indiferente ao risco, nenhuma alteração no nível do retorno de um ativo é exigida quando ocorre elevação do risco. No entanto, algumas pessoas são propensas ao risco, sendo atraídas pela sua elevação. Essa tendenciosidade leva o indivíduo a requerer menores retornos quando correm maiores riscos. Por sua vez, quando se é avesso ao risco, incrementos na taxa do retorno são exigidos na alta dos riscos; caso contrário, o ativo é desprezado, pois se exige compensação quando a possibilidade de perdas se eleva. A maioria das pessoas se enquadra nesse tipo de comportamento conservador e nãoagressivo (Gitman, 1997), preferindo ativos com maior relação retorno/ risco.

\subsection{Modelo analítico}

\subsubsection{Indicadores de viabilidade econômica}

A partir dos fluxos de caixa elaborados (Tabela 5 - Anexo 1), foram obtidos os indicadores econômicos para analisar a viabilidade econômica da produção de café não-irrigado e irrigado. Os indicadores utilizados foram: 
Valor Presente Líquido (VPL): representa o retorno monetário do investimento descontado o valor do dinheiro no tempo a uma taxa de desconto predeterminada ${ }^{7}$. Quando VPL $>0$, o projeto é economicamente viável. Em forma de equação:

$$
V P L=\sum_{t=0}^{n}(B-C)_{t} /(1+r)^{t}
$$

em que B são os benefícios; $\mathrm{C}$, os custos e investimentos gerados pelo projeto; $\mathrm{t}$, o período de tempo; $\mathrm{n}$, o tempo-limite; e r, a taxa de desconto predeterminada.

Taxa Interna de Retorno (TIR): é a taxa de desconto interna gerada pelo projeto que torna o VPL $=0$, considerando que os fluxos de caixa são reinvestidos pela própria TIR do projeto. Em forma de equação:

$$
V P L=\sum_{t=0}^{n}(B-C)_{t} /\left(1+r^{*}\right)^{t}=0
$$

em que B são os benefícios; $\mathrm{C}$, os custos e investimentos gerados pelo projeto; $\mathrm{t}$, o período de tempo; $\mathrm{n}$, o tempo-limite; e $\mathrm{r}^{*}$, a taxa de desconto interna (TIR). Quando a TIR é maior que a taxa de desconto predeterminada, o projeto é economicamente viável.

Taxa Interna de Retorno Modificada (TIRM): é um indicador que considera o custo de investimento e os juros de reinvestimento dos fluxos de caixa, sendo a TIRM a taxa de desconto que iguala o valor presente dos custos ao valor presente terminal. Em forma de equação:

\footnotetext{
A taxa de desconto adotada é de $9 \%$ ao ano, que representa a taxa de retorno do capital aplicado com correção monetária na caderneta de poupança.
} 


$$
\sum_{t=0}^{n} \frac{S C_{t}}{(1+r)^{t}}=\frac{\sum_{t=0}^{n} E C_{t}(1+k)^{n-t}}{(1+T I R M)^{n}}
$$

em que o termo à direita é o valor presente dos custos e o termo à esquerda é o valor presente terminal ou o valor futuro das entradas, que são reinvestidos ao custo de capital; SC, a saída de caixa; EC, a entrada de caixa; $t$, o período de tempo; $n$, o tempo-limite; e r, a taxa de desconto predeterminada. Quando a TIRM é maior que a taxa de desconto predeterminada, o projeto é economicamente viável.

Período de Payback (PP): é definido como o tempo de recuperação do capital investido. O PP é dado por:

$$
P P=\sum_{t=0}^{n}(B-C)_{t}=0
$$

em que B são os benefícios; $\mathrm{C}$, os custos e o investimentos gerados pelo projeto; $t$, o período de tempo; e $n$, o tempo-limite. Quanto menor o período de recuperação, maior a liquidez do projeto e maior a segurança do investidor.

\subsection{Análise de risco}

$\mathrm{Na}$ análise de risco, verificou-se preliminarmente a sensibilidade do poder de influência de cada variável, do fluxo de caixa das alternativas tecnológicas, isoladamente sobre os indicadores econômicos VPL e TIR, mediante utilização do software Excel. Para isso, considerou-se uma alta de $10 \%$ no preço de cada variável de entrada dos fluxos de caixa e, mantendo as demais variáveis constantes, avaliou-se o reflexo desse 
Alan Figueiredo de Arêdes, Maurinho Luiz dos Santos,

José Luis dos Santos Rufino \& Brício dos Santos Reis

aumento em termos percentuais sobre o VPL e TIR, variáveis de saída. No caso da variável produtividade, considerou-se uma alta de $10 \%$ no nível da produção.

Essa análise permitiu reconhecer as variáveis que provocam maior impacto sobre o retorno econômico das alternativas tecnológicas. São elas: preço do café, produtividade da lavoura, mão-de-obra eventual (temporária), fertilizante, fungicida e inseticida, uréia, energia elétrica, investimento na compra de terra e investimento em sistemas para irrigação por gotejamento e malha.

De posse dessas variáveis de entrada, utilizaram-se funções de probabilidade que representassem a melhor distribuição probabilística de cada variável, sendo escolhida a distribuição de probabilidade histograma para a variável preço do café e a distribuição triangular para as demais variáveis.

Após essa etapa, utilizou-se o método de simulação de Latin Hypercube, mediante o software @Risk, com mudança aleatória conjunta de todas as variáveis sensíveis dos fluxos de caixas com 10.000 interações, que foi o número suficiente para atingir a convergência das simulações e conferir credibilidade aos resultados. Esse método permitiu classificar e quantificar as variáveis que provocam maiores impactos sobre os indicadores de viabilidade econômica e obter a distribuição probabilística acumulada do retorno econômico.

\subsection{Fonte de dados}

Neste artigo, analisaram-se os resultados econômicos do emprego de cinco alternativas tecnológicas na produção de café, descritas resumidamente na Tabela 1 e Tabela 5 (Anexo 1). 
Tabela 1 - Quadro-resumo das cinco alternativas tecnológicas de produção de café

\begin{tabular}{lccccc}
\hline \multirow{2}{*}{ Parâmetro } & \multicolumn{5}{c}{ Alternativas tecnológicas } \\
\cline { 2 - 6 } & $\mathrm{A}$ & $\mathrm{B}$ & $\mathrm{C}$ & $\mathrm{D}$ & $\mathrm{E}$ \\
\hline Irrigação & Não & Não & Gotejamento & Gotejamento & Malha \\
Produtividade* & 25 & 42,5 & 65 & 65 & 65 \\
Adubação & Manual & Manual & Manual & Fertirrigação & Manual \\
* Referente à produção média, em sacas beneficiadas de 60 kg/ha, entre os anos 5 e 12 de \\
vida do cafezal. \\
Fonte: Elaborado pelo autor, de acordo com experimento realizado pelo DEA e consultas \\
a técnicos.
\end{tabular}

Os dados referentes às alternativas tecnológicas produtivas de café $\mathrm{B}$, $\mathrm{C}$ e D são oriundos de pesquisa realizada pelo Departamento de Engenharia Agrícola (DEA) da Universidade Federal de Viçosa (UFV) durante cinco anos - 1999/2004 - em uma área de 1,2 hectare na fazenda Laje, município de Viçosa/MG. O café estudado foi o Catuaí, com aproximadamente 11 anos e espaçamento de 3,1 x 1,0 m, com irrigação e fertirrigação parcial, sem irrigação nos meses de junho, julho e agosto, em função do déficit hídrico para otimização da floração.

O artigo incorporou ainda mais duas alternativas tecnológicas de produção: uma não-irrigada, com baixa produtividade da lavoura devido à pouca utilização de insumos e ao ineficiente manejo do cafezal, que corresponde à alternativa $\mathrm{A}$; e a outra tecnologia foi a $\mathrm{E}$, que corresponde ao café irrigado por malha. Os índices técnicos das duas alternativas foram construídos por meio de consultas a técnicos.

Considerou-se a implantação de uma lavoura de café e dos sistemas de irrigação no período zero e com a lavoura produzindo a primeira safra a partir do terceiro ano, tendo a vida útil produtiva de 15 anos. O preço do café adotado foi a média histórica de $\mathrm{R} \$ 297,00$ por saca, ocorrida entre janeiro de 1990 e dezembro de 2005, nas cinco alternativas, sendo o preço referente ao café arábica obtido na Cooperativa de Cafeicultores 
da Região de São Sebastião do Paraíso-MG (COOPARAÍSO), sendo os preços corrigidos pelo IGP-DI com base em dezembro de 2005. Para cada alternativa tecnológica de produção foram consideradas as produtividades em sacas beneficiadas de $60 \mathrm{~kg} / \mathrm{ha}$, como se observa na Tabela 2.

Tabela 2 - Produtividade dos cafezais nas diferentes alternativas tecnológicas de produção no município de Viçosa-MG

\begin{tabular}{ccccccc}
\hline Alternativas & \multicolumn{7}{c}{ Anos } \\
\cline { 2 - 7 } & 3 & 4 & $5-12$ & 13 & 14 & 15 \\
\hline A & 15 & 20 & 25 & 20 & 15 & 10 \\
B & 20 & 30 & 42,5 & 35 & 30 & 25 \\
C & 30 & 40 & 65 & 55 & 50 & 40 \\
D & 30 & 40 & 65 & 55 & 50 & 40 \\
E & 30 & 40 & 65 & 55 & 50 & 40 \\
\hline
\end{tabular}

Fonte: Elaborado pelo autor, de acordo com experimentos realizados pelo DEA e consultas a técnicos.

\section{Resultados e discussão}

Analisando os indicadores, com a ocorrência do preço médio do café igual a $\mathrm{R} \$ 297,00$ durante a vida útil das alternativas tecnológicas, constatou-se que a alternativa tecnológica mais indicada é a produção de café irrigada por malha, seguida pela fertirrigada e irrigada por gotejamento.

Com a utilização da taxa de desconto de $9 \%$ ao ano, que representa aproximadamente o retorno nominal do capital aplicado na caderneta de poupança, o produtor recuperou o capital investido nas cinco diferentes alternativas tecnológicas de acordo com o VPL, incrementando seu valor de mercado em $\mathrm{R} \$ 823,72$ na alternativa $\mathrm{A}$; $\mathrm{R} \$ 9.858,91$ na B; e R $27.217,19$ na E (Tabela 3). 
De acordo com o indicador TIR, todas as alternativas tecnológicas são economicamente viáveis, pois as TIRs foram superiores à taxa de desconto de $9 \%$ ao ano, sendo de $10,04 \%$ na alternativa A, $17,04 \%$ na B e $24,97 \%$ na E. Já pelo indicador TIRM, embora menor para todas as alternativas quando comparado à TIR, todas as alternativas também são viáveis, pois seus valores foram maiores que a taxa de desconto (Tabela 3 ).

O indicador PP também evidencia a superioridade econômica na geração de retornos do cafezal irrigado e fertirrigado, pois essas alternativas possibilitaram menor tempo de recuperação do capital investido, sendo de 8,39 anos na alternativa A, 6,89 anos na B e 5,68 anos na E (Tabela 3).

Tabela 3 - Indicadores de viabilidade econômica no cultivo de 1 ha de café não-irrigado, irrigado e fertirrigado, durante 15 anos de produção, em Viçosa-MG

\begin{tabular}{ccccccc}
\hline Indicador & Unidade & \multicolumn{5}{c}{ Alternativas tecnológicas } \\
\cline { 3 - 7 } & & $\mathrm{A}$ & $\mathrm{B}$ & $\mathrm{C}$ & $\mathrm{D}$ & $\mathrm{E}$ \\
\hline VPL & $\mathrm{R} \$$ & 823,72 & $9.858,91$ & $24.354,17$ & $25.030,45$ & $27.217,19$ \\
TIR & $\%$ & 10,04 & 17,04 & 21,66 & 22,04 & 24,97 \\
TIRM & $\%$ & 9,47 & 12,73 & 14,60 & 14,74 & 15,84 \\
PP & Anos & 8,39 & 6,89 & 6,07 & 6,02 & 5,68 \\
\hline
\end{tabular}

Fonte: Dados da pesquisa.

Pela análise de sensibilidade, a variável com maior poder de influência sobre o VPL é o preço do café, pois a elevação de $1 \%$ no preço provocou a elevação do VPL em $0,871 \%$ na alternativa tecnológica A, $0,911 \%$ na $\mathrm{B}, 0,928 \%$ na $\mathrm{C}, 0,929 \%$ na $\mathrm{D}$ e $0,927 \%$ na $\mathrm{E}$. A segunda variável com maior poder significativo de influência sobre o VPL é a produtividade, uma vez que um aumento de $1 \%$ nessa variável causou elevação entre $0,447 \%$ e $0,340 \%$ no VPL das alternativas tecnológicas. As demais variáveis tiveram pouca influência sobre o VPL, e seus sinais negativos encontrados indicam que elevações em seus valores diminuem o VPL (Tabela 4). 
Alan Figueiredo de Arêdes, Maurinho Luiz dos Santos,

José Luis dos Santos Rufino \& Brício dos Santos Reis

Tabela 4 - Análise da sensibilidade do VPL em relação às variáveis mais sensíveis do fluxo de caixa na produção de café nãoirrigado, irrigado e fertirrigado, em Viçosa-MG

\begin{tabular}{cccccc}
\hline Variável & \multicolumn{5}{c}{ Variação do VPL } \\
\cline { 2 - 6 } & $\mathrm{A}$ & $\mathrm{B}$ & $\mathrm{C}$ & $\mathrm{D}$ & $\mathrm{E}$ \\
\hline Preço do café & 0,871 & 0,911 & 0,928 & 0,929 & 0,927 \\
Produtividade & 0,447 & 0,379 & 0,340 & 0,343 & 0,343 \\
Mão-de-obra & $-0,015$ & $-0,009$ & $-0,006$ & $-0,005$ & $-0,007$ \\
Fertilizante & $-0,060$ & $-0,043$ & $-0,029$ & - & $-0,027$ \\
Fungicida e inseticida & - & $-0,016$ & $-0,007$ & $-0,008$ & $-0,009$ \\
Uréia & - & - & - & $-0,016$ & - \\
Energia & - & 0,000 & 0,000 & 0,000 & $-0,003$ \\
Água & 0,000 & 0,000 & 0,000 & 0,000 & $-0,005$ \\
Terra & $-0,057$ & $-0,033$ & $-0,024$ & $-0,022$ & $-0,021$ \\
Sistema Gotejamento & - & - & $-0,014$ & $-0,013$ & - \\
Sistema Malha & - & - & - & - & $-0,004$ \\
\hline
\end{tabular}

(-) Traços indicam que tal variável não é utilizada na alternativa tecnológica; logo, não existe um coeficiente para ela.

Fonte: Dados da pesquisa.

Quando analisado o risco pela distribuição acumulada da probabilidade de ocorrência do VPL, a uma taxa de desconto de $9 \%$ ao ano (Figura 2), as alternativas tecnológicas A e B concentram os menores retornos sob condições de risco em todos os níveis de probabilidade, ou seja, dada certa probabilidade de ocorrência do VPL, seus valores sempre foram menores para A e B. Além disso, ocorreu $46 \%$ de probabilidade de o VPL ser igual ou menor que zero na alternativa $A, 32 \%$ na $B$ e entre $15 \%$ e $19 \%$ na D, E e F. 


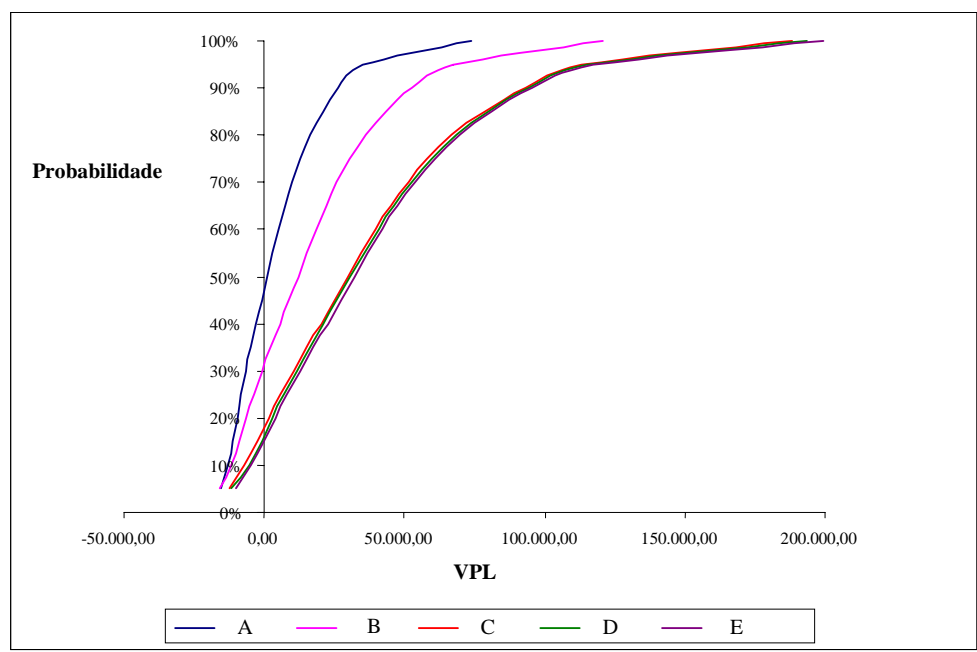

Figura 2 - Análise gráfica de risco pela distribuição acumulada da probabilidade de ocorrência do VPL no cultivo de café nãoirrigado, irrigado e fertirrigado, em Viçosa-MG.

Fonte: Dados da pesquisa.

Pela análise de risco através da distribuição acumulada da probabilidade de ocorrência da TIR, evidenciou-se a superioridade das alternativas tecnológicas irrigada e fertirrigada sobre a cafeicultura não-irrigada, havendo entre $35 \%$ e $40 \%$ de possibilidade de a TIR na alternativa A ser menor que a taxa de desconto de $9 \%$ e entre $25 \%$ e $30 \%$ de a TIR ser menor que a taxa de desconto na alternativa B. A alternativa tecnológica $\mathrm{C}$ e a $\mathrm{D}$ apresentaram entre $15 \%$ e $20 \%$ de probabilidade de gerar uma TIR inferior à taxa de desconto; e a alternativa E, entre $10 \%$ e $15 \%$ (Figura 3 ).

Da mesma forma que o VPL, para qualquer nível de probabilidade a TIR é menor nas alternativas não-irrigadas. Por exemplo, tomando-se o nível de $60 \%$ de probabilidade, a TIR da alternativa tecnológica A foi de no máximo $15,99 \%$ ao ano e, na alternativa $\mathrm{B}$, no máximo de $21,83 \%$, contra $25,60 \%$ na C, $26,30 \%$ na D e $28,65 \%$ na E (Figura 3 ). 


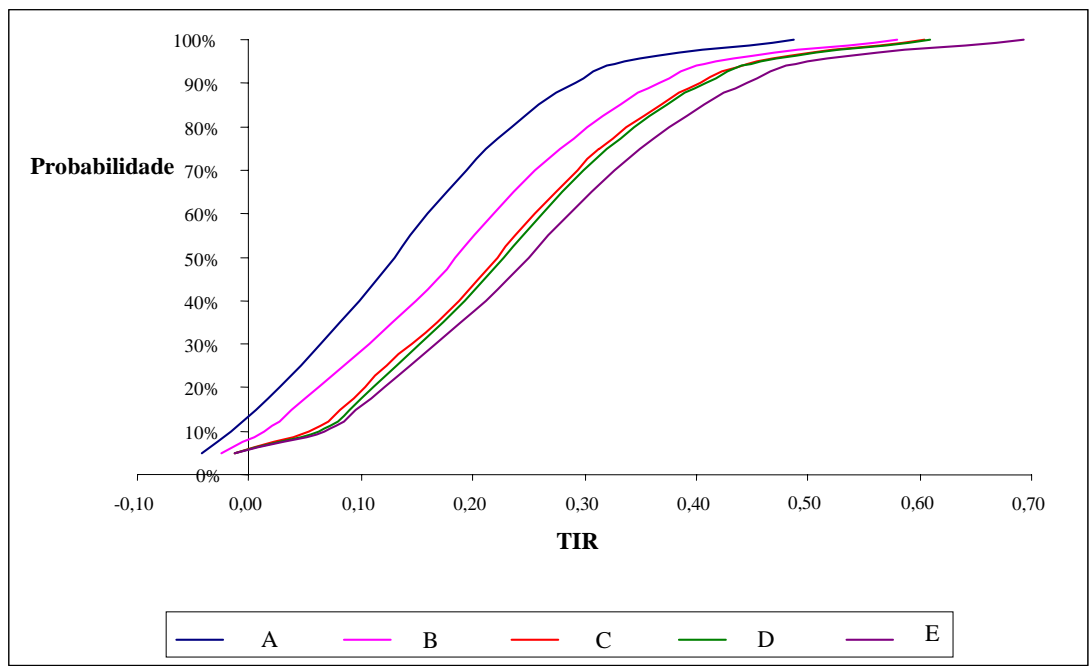

Figura 3 - Análise gráfica de risco pela distribuição acumulada da probabilidade de ocorrência da TIR no cultivo de café nãoirrigado, irrigado e fertirrigado, em Viçosa-MG.

Fonte: Dados da pesquisa.

\section{Conclusões}

Diante do problema relativo à viabilidade econômica da utilização da irrigação em regiões tradicionais, com clima úmido favorável à produção de café, este trabalho teve por objetivo analisar a viabilidade econômica do emprego de alternativas tecnológicas de irrigação na produção de café na região de Viçosa-MG, tendo como hipótese o fato de que os benefícios líquidos gerados a partir da implantação das alternativas tecnológicas com sistemas de irrigação são positivos e maiores que os oriundos da cafeicultura não-irrigada.

De acordo com os resultados, a cafeicultura irrigada possui os melhores resultados econômicos, sendo os indicadores de viabilidade VPL, TIR, TIRM e PP mais favoráveis à produção irrigada por malha, seguida pela fertirrigada e irrigada por gotejamento. 
Pela análise de risco, independentemente da alternativa tecnológica de produção (não-irrigada, irrigada ou fertirrigada), todas são sensíveis em seus retornos econômicos em relação às variações do preço do café e da produtividade da lavoura. Entretanto, as alternativas tecnológicas são pouco sensíveis às variações nos preços de mão-de-obra eventual, fertilizante, uréia, energia elétrica, água, fungicida e inseticida, de aquisição da terra e dos sistemas de irrigação e fertirrigação por gotejamento e malha, o que permite concluir que o principal risco que atinge o retorno econômico do cafezal é causado pela variação do preço do café e da produtividade.

Em relação à probabilidade de ocorrência do VPL e TIR, as alternativas tecnológicas menos arriscadas são a produção irrigada por malha e a fertirrigada e irrigada por gotejamento, respectivamente, uma vez que apresentaram as menores probabilidades de fracasso, ou seja, de gerar VPL negativo e TIR inferior à taxa de desconto.

Conclui-se que a cafeicultura irrigada é rentável e superior à alternativa tecnológica de produção não-irrigada mesmo em regiões tradicionalmente produtoras, como a de Viçosa-MG, caracterizada por condições hídricas favoráveis à cultura do café, pois, além de elevar o retorno econômico, ainda reduz o risco da atividade.

\section{Referências}

BUARQUE, C. Avaliação econômica de projetos: uma apresentação didática. 8. ed. Rio de Janeiro: Campus, 1991. 266 p.

COELHO, G.; SILVA, A. M.; SILVA, P. A. M.; LIMA, E. P. Custo de produção da cafeicultura irrigada. Disponível em: <http:// www.editora.ufla.br/revista/suple_2002/art21.pdf >. Acesso em: 20 jan. 2006.

DEBERTIN, D. L. Agricultural production economics. New York: Collier Macmillan Canada, Inc., 1986. 366p. 
Alan Figueiredo de Arêdes, Maurinho Luiz dos Santos,

José Luis dos Santos Rufino \& Brício dos Santos Reis

GITMAN, L. J. Princípios de administração financeira. 7. ed. São Paulo: Harbra, 1997.

IRRIGAÇÃO E TECNOLOGIA MODERNA (ITEM). Um panorama da cafeicultura irrigada no Brasil. Brasília: Associação Brasileira de Irrigação e Drenagem (ABID), n. 48, set. 2000.

MANTOVANI, E. C. Cafeicultura irrigada: produtividade, rentabilidade com sustentabilidade. In: MANTOVANI, E. C.; SOARES, A. R. Irrigação do cafeeiro: informações técnicas e coletânea de trabalhos. Viçosa: UFV, DEA, 2003. p. 9-45.

PINDYCK, R. S.; RUBINFELD, D. L. Microeconomia. 6. ed. São Paulo: Pearson Prentice Hall, 2006. 641p.

REZENDE, J. L. P.; OLIVEIRA, A. D. Análise econômica e social de projetos florestais. Viçosa: Editora UFV, 2001. 389 p.

ROSS, S. A.; WESTERFIELD, R. W.; JORDAN, B. D. Princípios de administração financeira. 2. ed. São Paulo: Atlas, 1998. 425 p.

SILVA, A. M.; SILVA, R. A.; COELHO, G.; OLIVEIRA, P. M.; SILVA, A. C.; SATO, F. A. Efeito da época de irrigação e do parcelamento de adubação sobre a produtividade do cafeeiro (safra 01/02). In: SIMPÓSIO BRASILEIRO DE PESQUISA EM CAFEICULTURA IRRIGADA, 6., 2003, Araguari. Anais... Uberlândia: UFU, 2003. p. 75-79.

THIRY-CHERQUES, H. R. Modelagem de projetos. 2. ed. São Paulo: Atlas, 2004. 265 p.

VARIAN, H. R. Intermediate microeconomics: a modern approach. 3. ed. New York: W. W. Norton e Company, 1993. 623p.

WOILER, S.; MATHIAS, W. F. Projetos: planejamento, elaboração e análise. São Paulo: Atlas, 1996. 294 p. 


\section{Anexo 1}

Tabela 5 - Fluxos de caixa (em R \$) para implantação e manutenção por 15 anos das diferentes alternativas tecnológicas de produção de café em 1 ha, para o município de Viçosa-MG

\begin{tabular}{|c|c|c|c|c|c|c|c|}
\hline \multicolumn{8}{|c|}{ Alternativa tecnológica A } \\
\hline Descrição & Ano 0 & Ano 1 & Ano 2 & Ano 3 & Ano 4 & $\ldots$ & Ano 15 \\
\hline A- Receita & & & 4455,00 & 5940,00 & 7425,00 & $\ldots$ & 2970,00 \\
\hline B- Custos & & 2967,72 & 4901,96 & 5204,14 & 5496,70 & $\ldots$ & 4673,66 \\
\hline C- Lucros tributáveis & & $-2967,72$ & $-446,96$ & 735,86 & 1928,30 & $\ldots$ & $-1703,66$ \\
\hline E- Lucro líquido após IR & & $-2967,72$ & $-446,96$ & 533,50 & 1398,02 & $\ldots$ & $-1703,66$ \\
\hline F- Deprec. e restituição do IR & & 346,11 & 663,11 & 1035,11 & 1035,11 & $\ldots$ & 663,11 \\
\hline G- Investimentos & $-9843,72$ & & & & & $\ldots$ & \\
\hline H- Fluxo líquido de caixa & $-9843,72$ & $-2621,61$ & 216,16 & 1568,61 & 2433,13 & $\ldots$ & $-540,55$ \\
\hline \multicolumn{8}{|c|}{ Alternativa tecnológica B } \\
\hline Descrição & Ano 0 & Ano 1 & Ano 2 & Ano 3 & Ano 4 & $\ldots$ & Ano 15 \\
\hline A- Receita & & & 5940,00 & 8910,00 & 12622,50 & $\ldots$ & 7425,00 \\
\hline B- Custos & & 4299,50 & 6567,70 & 7122,82 & 7876,71 & $\ldots$ & 6886,00 \\
\hline C- Lucros tributáveis & & $-4299,50$ & $-627,70$ & 1787,18 & 4745,79 & $\ldots$ & 539,00 \\
\hline E- Lucro líquido após IR & & $-4299,50$ & $-627,70$ & 1295,71 & 3440,69 & $\ldots$ & 390,77 \\
\hline F- Deprec. e restituição do IR & & 360,00 & 667,00 & 1049,00 & 1049,00 & $\ldots$ & 1049,00 \\
\hline G- Investimentos & $-11425,5$ & & & & & $\ldots$ & \\
\hline H- Fluxo líquido de caixa & $-11425,5$ & $-3939,50$ & 49,30 & 2344,71 & 4489,69 & $\ldots$ & 1939,77 \\
\hline \multicolumn{8}{|c|}{ Alternativa tecnológica $\mathrm{C}$} \\
\hline Descrição & Ano 0 & Ano 1 & Ano 2 & Ano 3 & Ano 4 & $\ldots$ & Ano 15 \\
\hline A- Receita & & & 8910,00 & 11880,00 & 19305,00 & $\ldots$ & 11880,00 \\
\hline B- Custos & & 5201,72 & 8118,60 & 8673,72 & 10151,51 & $\ldots$ & 8763,72 \\
\hline C- Lucros tributáveis & & $-5201,72$ & 791,39 & 3206,27 & 9153,49 & $\ldots$ & 3116,27 \\
\hline E- Lucro líquido após IR & & $-5201,72$ & 573,76 & 2324,55 & 6636,28 & $\ldots$ & 2259,30 \\
\hline F- Deprec. e restituição do IR & & 651,67 & 1340,67 & 1340,67 & 1340,67 & $\ldots$ & 1340,67 \\
\hline G- Investimentos & $-17577,7$ & & & & & $\ldots$ & \\
\hline H- Fluxo líquido de caixa & $-17577,7$ & $-4550,06$ & 1914,43 & 3665,22 & 7976,95 & $\ldots$ & 4099,97 \\
\hline \multicolumn{8}{|c|}{ Alternativa tecnológica D } \\
\hline Descrição & Ano 0 & Ano 1 & Ano 2 & Ano 3 & Ano 4 & $\ldots$ & Ano 15 \\
\hline
\end{tabular}


Alan Figueiredo de Arêdes, Maurinho Luiz dos Santos,

José Luis dos Santos Rufino \& Brício dos Santos Reis

\begin{tabular}{|c|c|c|c|c|c|c|c|}
\hline A- Receita & & & 8910,00 & 11880,00 & 19305,00 & $\ldots$ & 11880,00 \\
\hline B- Custos & & 5106,43 & 8023,30 & 8578,42 & 10056,21 & $\ldots$ & 8668,42 \\
\hline C- Lucros tributáveis & & $-5106,42$ & 886,69 & 3301,57 & 9248,79 & $\ldots$ & 3211,58 \\
\hline E- Lucro líquido após IR & & $-5106,42$ & 642,85 & 2393,64 & 6705,37 & $\ldots$ & 2328,39 \\
\hline F- Deprec. e restituição do IR & & 651,67 & 1340,67 & 1340,67 & 1340,67 & $\ldots$ & 1340,67 \\
\hline G- Investimentos & $-17482,4$ & & & & & $\ldots$ & \\
\hline H- Fluxo líquido de caixa & $-17482,4$ & $-4454,76$ & 1983,52 & 3734,31 & 8046,04 & $\ldots$ & 4169,06 \\
\hline \multicolumn{8}{|c|}{ Alternativa tecnológica $\mathrm{E}$} \\
\hline Descrição & Ano 0 & Ano 1 & Ano 2 & Ano 3 & Ano 4 & $\ldots$ & Ano 15 \\
\hline A- Receita & & & 8910,00 & 11880,00 & 19305,00 & $\ldots$ & 11880,00 \\
\hline B- Custos & & 5062,84 & 7979,72 & 8534,83 & 10012,62 & $\ldots$ & 8624,83 \\
\hline C- Lucros tributáveis & & $-5062,84$ & 930,28 & 3345,17 & 9292,38 & $\ldots$ & 3255,17 \\
\hline E- Lucro líquido após IR & & $-5062,84$ & 674,46 & 2425,25 & 6736,97 & $\ldots$ & 2360,00 \\
\hline F- Deprec. e restituição do IR & & 462,78 & 1151,78 & 1151,78 & 1151,78 & $\ldots$ & 1151,78 \\
\hline G- Investimentos & $-14038,8$ & & & & & $\ldots$ & \\
\hline H- Fluxo líquido de caixa & $-14038,8$ & $-4600,06$ & 1826,23 & 3577,02 & 7888,75 & $\ldots$ & 4011,77 \\
\hline
\end{tabular}

Fonte: Dados de pesquisa.

Abstract — The objective of this paper is analyze the economic viability of implanting different technological alternatives of irrigation in the culture of coffee in the municipal district of Viçosa-MG. Technical and financial parameters of coffee cultivation were analyzed in five different technological alternatives: (A) - no-irrigated production with low productivity, (B) - no-irrigated production with high productivity, (C) - irrigated production by leaking, (D) - irrigated production by leaking fertilizing, (E) - irrigated production by mesh. For each one of there alternatives, Present Value, Internal Rate of Return, the Rate Interns of Return Modified and the Payback Period, being made the risk simulations were performed using the Latin Hypercube method. Results showed that the alternatives of production white irrigated were economically superior to the alternatives without no-irrigated, even in traditional producers areas, as the municipal district of Viçosa-MG. In addition to elevating the economical return and reducing the of the Payback Period capital invested, irrigated coffee reduces the risk of the activity.

Keywords: coffee, return, risk. 
REVISTA DE ECONOMIA E AGRONEGÓCIO, VOL.5, $N^{\circ} 2$ 